\section{Extinction as a function of size of goal box, reinforcement schedule, and competing}

\section{responses}

H. WILLARD BRADLEY, York University, Toronto 12, Canada, and RODERICK WONG, ${ }^{2}$ University of British Columbia, Vancouver 168, Canada

Forty-eight Ss were assigned randomly to one of four acquisition conditions and were trained and tested in a straight-alley runway. The acquisition conditions were: continuous reinforcement in a large goalbox, continuous reinforcement in $a$ small goalbox, partial reinforcement in a large goalbox, and partial reinforcement in a small goalbox. Following completion of the 60 acquisition trials, each $S$ received 50 nonreward trials that were given in blocks of five trials per day. On both acquisition and extinction trials, runway retracing was recorded, and time measures were taken for startbox and nunway performance. The results indicate that $S$ s trained on a noncontinuous reinforcement schedule while running to a large goalbox exhibit fewer competing responses and a greater resistance to extinction. The results are discussed with regard to a competing-response interpretation of the partial-reinforcement effect.

In their test of the competing response theory of extinction, Adelman \& Maatsch (1955) have shown that the type of response elicited by nonreward can influence an animal's resistance to extinction of a running response acquired under a continuous reinforcement (CRF) schedule. More recently, Kirkpatrick, Pavlik, \& Reynolds (1964) extended this analysis to the partial-reinforcement (PRF) situation. The aim of the present study is to replicate, in principle, the Kirkpatrick et al experiment and to examine the status of competing-response theory.

According to a competing-response interpretation, it is predicted that Ss on a CRF schedule would exhibit few competing or nonfunctional responses during the acquisition trials compared with those on a PRF schedule. Because of the few nonfunctional responses elicited in the CRF condition, there should be little opportunity for such responses to be extinguished, dropped out, or modified during acquisition. However, the condition of nonreward during extinction would elicit competing responses, and these, in turn, would interfere with the running response. In contrast, in the PRF CRF rats. condition, competing responses would be elicited from $S s$ during the nonrewarded trials during acquisition and, with subsequent adaptation to this condition, Ss would have an opportunity to modify their nonfunctional responses. Support for this hypothesis may be found in Weinstock's (1954) observation that rats on a PRF schedule tend to adapt out the agitated behavior that formerly was elicited by cues in the empty goalbox. Hence, when extinction began, they made fewer responses that competed with the instrumental running response than did the

The other variable that Kirkpatrick et al (1964) found to affect the degree of competing responses is the size of the goalbox (GB). A large GB is more likely to elicit gross intra-GB movements from $\mathrm{Ss}$ than a smaller box. It follows that such intra-GB responses are more compatible with the preceding running activity than a lesser degree of such movements. For this reason, Ss running to a small GB are more likely to exhibit faster extinction than are those running to a large GB, because of the greater compatibility of large-box movements and running behavior.

The relevance of the size of the GB to resistance to extinction has recently been demonstrated by Mikulka, Vogel, \& Spear (1967). They found that Ss given postconsummatory delay in a large GB showed faster goal speeds during extinction trials in the runway than those given postconsummatory delay in a small box. Mikulka et al hypothesized that the nonreward cues in the GB were being conditioned to the instrumental foodcup response and that, subsequently, these cues maintain performance during extinction. They also suggest that the more similar the postconsummatory delay-goal responses are to the instrumental-goal response, the stronger this conditioning should be. A large GB would increase the similarity between the foodcup-approach response and the instrumental-running response. A smaller GB would minimize the similarity of such responses.

The present experiment consisted of training the animals to run to either a large or a small GB while rewarded on either a CRF or a PRF schedule in a straight alleyway. Essentially, the design is similar to that used by Kirkpatrick et al (1964), except that the rats were given a greater number of extinction trials in the present experiment. The reason for this procedure is presented in the discussion section.

Following the Kirkpatrick et al results, it was expected that rats running to a large GB on a $50 \%$ schedule would show greater resistance to extinction than would those trained under the other conditions. Moreover, it is predicted that the magnitude of the partial-reinforcement effect (PRE) would be greater under the large-GB condition than under the small-GB condition.

\section{SUBJECTS}

The Ss were 48 female albino rats of the Wistar strain. They were about 90 days old at the beginning of the experiment. APPARATUS

The apparatus was a 25-in. straight-alley runway, connecting a 12-in. startbox (SB) with the GB. The width and height of both the runway and SB were $3 \times 6$ in. The large GB measured $12 \times 12 \times 12$ in., while the small $G B$ was $3 \times 12 \times 6$ in. Guillotine doors separated the alley from both the SB and the GB. The apparatus was painted grey, and the top of the runway, startbox, and goalboxes consisted of hardware cloth that was hinged to the sides of the apparatus. Metal food dishes, measuring 2 in. in diam and $1 / 4$ in. in height, were placed in each of the goalboxes.

Starting and running times were measured with $.01-\mathrm{sec}$ electric timers that were activated by photocells. When the guillotine door of the SB was lifted, the first timer was activated. When $S$ broke the light beam by crossing 6 in. from the SB, the first timer was stopped and the second timer was automatically started. The second timer was stopped when S broke the second light beam, which was 23 in. from the SB door and 2 in. from the GB. PROCEDURE

Four days prior to the experiment, Ss were placed on a feeding schedule of $10 \mathrm{~g}$ of Purina Chow per day and were maintained on this schedule throughout the experiment. Water was available in the home cage at all times. Each $S$ was handled $5 \mathrm{~min}$ per day and allowed to explore the apparatus for $10 \mathrm{~min}$ each day during the 4-day period. During pretraining, Ss were assigned randomly to one of the following conditions during acquisition: (1) $50 \%$ reward and large GB (50-L), (2) $50 \%$ reward and a small GB (50-S), (3) $100 \%$ reward and a large GB (100-L), and (4) $100 \%$ reward and a small GB (100-S). Following handling and habituation, Ss were given their pretraining.

Pretraining consisted of 2 days in which all Ss received five reinforced trials per day with an intertrial interval (ITI) of about $15 \mathrm{~min}$. If $\mathrm{S}$ completed two consecutive trials with a total running time of less than 


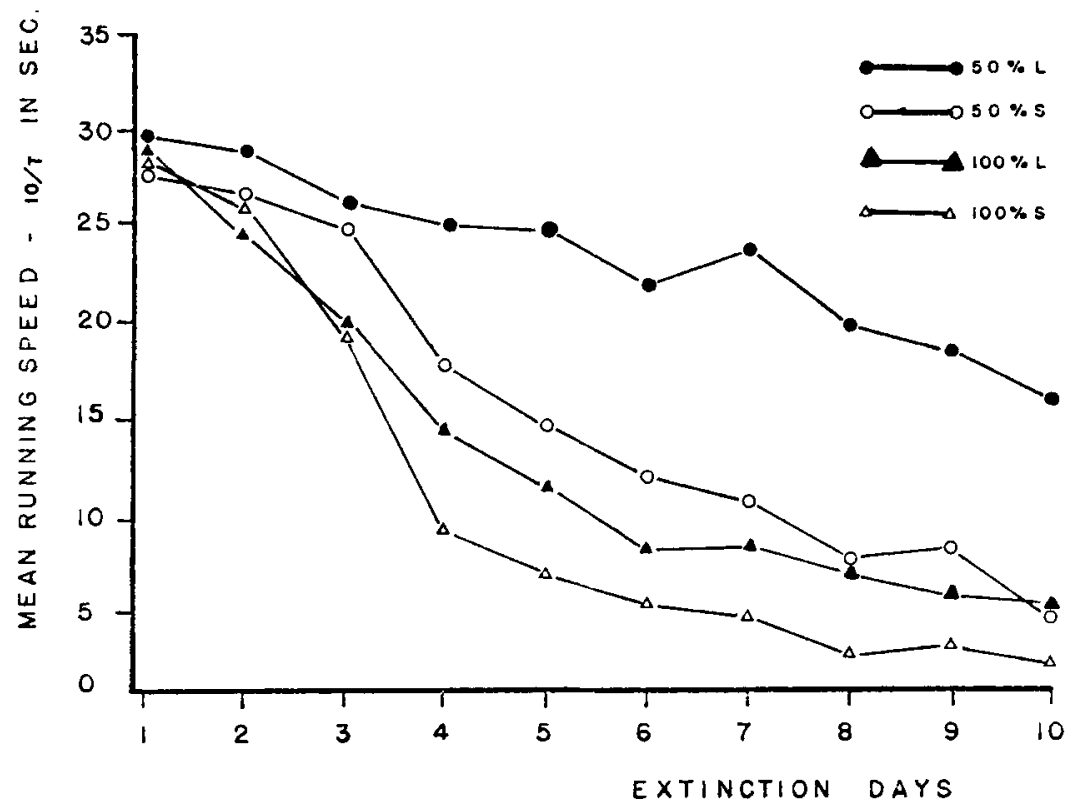

$2 \mathrm{~min}$, pretraining was discontinued for it. Immediately following pretraining, each $S$ was given 60 acquisition trials ( 6 trials per day for 10 consecutive days). The Ss ran to either a large or a small GB while on either a $50 \%$ or a $100 \%$ reinforcement schedule, depending on their group assignment. On all trials, each $S$ was detained in the GB for a period of $20 \mathrm{sec}$ and received three $45-\mathrm{mg}$ Noyes pellets on either a $50 \%$ or a $100 \%$ schedule. The ITI was $15 \mathrm{~min}$. After the start door was raised, both starting and running times were taken.

Upon completion of the acquisition trials, each $S$ received 50 extinction trials at a rate of 5 trials per day, with a $15-\mathrm{min}$ ITI. If $S$ failed to reach the GB within $3 \mathrm{~min}$, it was removed from the apparatus and the score was recorded as $3 \mathrm{~min}$.

In addition to noting the starting and running times, a record was kept of the incidence of competing responses emitted by each $S$ during the extinction trials (Mandler, 1962). During each trial, E noted whether $S$ stopped running in the alleyway or whether it retraced. The criterion for retracing was that $S$ took five steps back to the SB after it had interrupted the first photobeam.

\section{RESULTS}

All time measures were converted into speed scores (10/lat), and a mean speed score was computed for each block of five trials. Although there was a significant days (trials) effect during acquisition, analysis of variance indicated that the groups did not differ in either starting or running speed. However, the analysis of the extinction trials indicated significant main as well as interaction effects. Since starting and running speeds provided similar results, under the small GB condition.

\section{DISCUSSION}

Fig. 1. Mean running speeds for each day of extinction.

running during extinction. It appears that the greatest resistance to extinction occurs under conditions that are most conducive to enabling $S$ to adapt to the interfering effects of nonfunctional responses during acquisition.

Further support for the competing-response interpretation comes from the fact that the magnitude of the PRE was greater in the groups trained under the large-GB condition than it was in those trained under the small-GB condition. Although the data of Kirkpatrick et al (1964) was interpreted in a manner similar to the above, they did not find a significant interaction between GB size and reinforcement schedule. It is believed that the significant interaction observed in the present experiment was due to the fact that Ss received a greater number of extinction trials (50) than did those in the Kirkpatrick et al study (30). Whereas Kirkpatrick et al gave their Ss 10 massed extinction trials for 3 days, the present Es used 5 trials with a 15-min ITI per day over a 10-day period. Also, the large goalbox in the present experiment was 6 in. higher than that used in the Kirkpatrick et al study. The height of the box was chosen in order to allow the animals to stand fully erect on their hind legs once they were in the GB.

The results on the other dependent variable, amount of retracing during extinction, also support the predictions made earlier. Consistent with Jones \& Bridge's (1966) findings, not only did the PRF animals run faster during extinction than those under CRF, but they also exhibited fewer competing responses during these trials. The present study also indicated that GB size affects the amount of competing responses elicited in the runway during extinction. Group 100-S emitted more competing responses during extinction than did Group 50-S. In general, these results show that regardless of GB size, Ss trained under a $50 \%$ reward schedule exhibited fewer competing responses than those trained under a $100 \%$ schedule. Also, when reward schedule was held constant, Ss trained under the large GB condition were more resistant to extinction than those trained

The data is consistent with the Kirkpatrick et al (1964) findings in showing that greatest resistance to extinction arises when $S$ is trained on a PRF schedule while running to a large GB. This finding supports the prediction that the type of response elicited during acquisition has an effect on the rat's

\section{REFERENCES}

ADELMAN, H., \& MAATSCH, J. Resistance to extinction as a function of the type of response elicited by frustration. Joumal of Experimental Psychology, 1955, 59, 61-65.

JONES, E. C., \& BRIDGES, C. C. Competing responses and the partial reinforcement effect. Psychonomic Science, 1966, 6, 483-484.

KIRKPATRICK, D. R., PAVLIK, W. B., \& REYNOLDS, W. F. Partial reinforcement extinction effect as a function of the size of the goal box. Joumal of Experimental Psychology, 1964, 68, 515-516.

MANDLER, G. The interruption of behavior. In D. Levine (Ed.), Nebraska Symposium on Motivation. Lincoln: University of Nebraska Press, 1962.

MIKULKA, P. J. VOGEL, J., \& SPEAR, N. E. Post-consummatory delay and goal box 
confinement. Psychonomic Science, 1967, 9, 381-382.

WEINSTOCK, S. Resistance to extinction of a running response following partial reinforcement under widely spaced trials. Journal of Comparative \& Physiological Psychology, 1954, 47, 318-323.

\section{Induced phenylketonuria (PKU): Lack of a critical period in development}

\begin{abstract}
ROBERT L. SCHALOCK, Hastings College, Hastings, Nebr. 68901
\end{abstract}

Thirty-six neonatal albino rats were intubed twice daily for 30 consecutive days under one of six L-phenylalanine or saline conditions to determine if there was a critical period in the development of their learning ability that was most sensitive to $L$-phenylalanine induced toxicity. Each $S$ was tested on the Maier reasoning test and on DRL:12 operant schedule beginning at 50 days of age. Results indicated that while no critical period was found, there was a larger learning deficit in Ss treated earlier and longer.

A number of recent studies dealing with the artificial induction of phenylketonuria (PKU) or hyperphenylaninemia ${ }^{2}$ in rats have suggested that there might be a critical period during which the organism is most sensitive to the effects of the excess L-phenylalanine. Goldstein (1965), for example, reports that the rate of B-Phenyllactate oxidation is slower in preweanling than in postweanling rats. Although Polidora, Cunningham, \& Waisman (1966a) were unable to detect a critical period in the development of PKU, they do report that the initial insult might occur sometime within the first 25 days of administration. Similarly, Schalock \& Klopfer (1967b) have reported an induced learning deficit that is larger in rats treated with excess L-phenylalanine from birth to 60 days, than in Ss treated from 31-60 days of age. The purpose of the present study was to determine whether, within the first 30 days of a rat's life, there is a critical period in the development of the S's leaming ability that is most sensitive to the effects of excess L-phenylalanine treatment. SUBJECTS

The Ss were 36 ( 19 male, 17 female) albino rats (Long-Evans strain) from the colony at Hastings College. The six experimental groups, comprising five litters,
NOTES

1. Based on an MA thesis done at University of British Columbia and supported in part by Grant APA-247 from the National Research Council of Canada to $R$. Wong.

2. Now at Windham College, Putney, Vt. 05346. were initially balanced with respect to sex, litter mates, and body weight.

\section{INTUBING PROCEDURE}

Within $24 \mathrm{~h}$ after birth, the Ss were divided into the following six groups: (1) Days 1-10 L-phenylalanine (L-ph), Days 11-30 saline (S); (2) 1-10 S, 11-20 L-ph, 21-30 S; (3) 1-20 L-ph, 21-30 S; (4) 1-20 S, 21-30 L-ph; (5) 1-30 L-ph; and (6) 1-30 S. Ss within each group were intubed twice daily ( 8 a.m. and 8 p.m.) with either L-phenylalanine $(3 \mathrm{~g} / \mathrm{kg}$ of a $5 \%$ L-phenylalanine solution $)^{3}$ or physiological saline $(60 \mathrm{cc} / \mathrm{kg})$. All intubation was by means of a polyethylene tube connected to a $20 \mathrm{ml}$ syringe. Weekly blood and urine samples were collected 105-150 min after intubation for serum-phenylaline ${ }^{4}$ and phenylpyruvic acid $^{5}$ determinations. A 20-day recovery period (Perez, 1965; Schalock \& Klopfer, 1967b) followed the 30-day intubation period.

APPARATUS AND

The Maier reasoning test (Maier, 1932) and a DRL (differential reinforcement of low rate of responding) 12 -sec schedule (Leaf, Carlton, \& Hess, 1965) conducted in a Gerbrands G 2110 operant conditioning unit were used to assess the S's learning ability. Half of the Ss in each group were tested first on the reasoning test and second on the DRL: 12 schedule, with the converse for the second group. All Ss were tested beginning at 50 days of age, and all were
TESTING PROCEDURES

maintained on a 23-h food-deprivation schedule during both tests.

The procedure for the reasoning test was the same as previously used (Schalock \& Klopfer, 1967b). The test employed three tables $(77 \mathrm{~cm}$ high), connected by three elevated runways $(132 \mathrm{~cm}$ long and $4 \mathrm{~cm}$ wide). Each table differed either in size, albedo, or floor texture from the other two. Animals were adapted to the apparatus by $1 / 2$-h exploratory periods on 8 successive days with no food present. On each of the next 18 days, each animal was placed on one of the tables with six food pellets. The feeding table varied from day to day in a random fashion, with the restriction that the animal was fed on each table six times in 18 days. When the pellets were partially consumed, the rat was moved to one of the other tables and permitted to run once either to the table with food or to the one without. After the first day, the erroneous response consisted in running to the table on which the animal had been fed on the previous day. Reasoning scores were the ratios of the difference in frequencies of correct and incorrect responses to the total number of responses. In addition, the amount of time spent at the choice point (a point equidistant from each of the three tables) was recorded for both correct and incorrect responses. The number of tables visited during adaptation and testing was also recorded.

The procedure for the DRL: 12 schedule involved six stages: (1) an initial training period on a FR 1:1 schedule until each $S$ was responding at least 110 times within a 30-min test period; (2) a 1:1 schedule for $10 \mathrm{~min}$ (10 reinforcements), followed by a 60 min extinction period; (3) a 30 -min $1: 1$ session during which all responses were reinforced; (4) a final 1:1 schedule involving 25 rewards; (5) the six DRL: 12 sessions $(50$ reinforcements per session); and (6) a final 45-min extinction period. During the six consecutive-day DRL:12 sessions, only those responses that occurred after a 12-sec period of not responding were reinforced. The S's score was the number of responses emitted to acquire the 50 reinforcements.

\section{RESULTS}

Biochemically, the Ss were phenylketonuric while receiving the excess

Table 1

Reasoning Scores Obtained on Maier Reasoning Test and Time Spent at Choice Point ( $N=6$ per group)

\begin{tabular}{cccc} 
& $\begin{array}{c}\text { Mean } \\
\text { Reasoning } \\
\text { Score }\end{array}$ & & \multicolumn{2}{c}{\begin{tabular}{c} 
Average Time (in sec) Spent at Choice Point \\
\cline { 3 - 4 }
\end{tabular}} & $\begin{array}{c}\text { On Correct } \\
\text { Response Trials }\end{array}$ & $\begin{array}{c}\text { On Incorrect } \\
\text { Response Trials }\end{array}$ \\
\hline $1-10$ L ph & $.11 \pm .07$ & 2.2 & 3.1 \\
1.20 L ph & $.04 \pm .10$ & 2.7 & 3.3 \\
$1-30$ L ph & $.02 \pm .13$ & 3.1 & 2.5 \\
$11-20$ L ph & $.04 \pm .09$ & 2.1 & 2.4 \\
$21-30$ L ph & $.20 \pm .12$ & 2.3 & 3.0 \\
$1-30$ Saline & $.55 \pm .08$ & 1.3 & 2.5 \\
\hline
\end{tabular}

